\title{
Pattern of Weight Loss after Successful Enucleation of an Insulin-producing Pancreatic Neuroendocrine Tumor
}

\author{
Mark Anthony Santiago Sandoval, 1,2 Tom Edward Ngo Lo, ${ }^{1}$ A'Ericson Berberabe, ${ }^{3}$ \\ Mark Anthony De Lusong, ${ }^{4}$ Juan Maria Ibarra $\mathrm{Co}^{5}$ \\ ${ }^{1}$ Section of Endocrinology, Diabetes and Metabolism, Department of Medicine, Philippine General Hospital \\ ${ }^{2}$ Department of Physiology, Philippine General Hospital \\ ${ }^{3}$ Division of Hepatobiliary and Pancreatic Surgery, Department of Surgery, Philippine General Hospital \\ ${ }^{4}$ Section of Gastroenterology, Department of Medicine, Philippine General Hospital \\ ${ }^{5}$ Section of Endocrinology, Diabetes and Metabolism, Department of Internal Medicine, Cardinal Santos Medical Center, San Juan City, Philippines
}

\begin{abstract}
We report the case of a patient with hypoglycemic symptoms and weight gain. Biochemical investigations revealed endogenous hyperinsulinemic hypoglycemia. A CT scan and MRI of the abdomen were initially not successful in localizing a pancreatic mass. However, an endoscopic ultrasound was able to demonstrate a pancreatic head mass. Enucleation of the mass resulted in clinical and biochemical improvement. This report also demonstrates the pattern of weight loss after surgery, showing an initial phase of gradual weight loss followed by a rapid loss of weight. This pattern of weight loss after successful removal of an insulin-producing pancreatic neuroendocrine tumor is a novel addition to the existing knowledge we have about this condition.
\end{abstract}

Key words: hypoglycemia, insulinoma, pancreatic neuroendocrine tumor, weight loss

\section{INTRODUCTION}

Insulinomas are rare tumors that produce excessive amounts of insulin causing hypoglycemia and weight gain. These tumors are now more appropriately called insulin-producing pancreatic neuroendocrine tumors (PNET). ${ }^{1}$ This case demonstrates the approach to diagnosis of a patient with hypoglycemia and the eventual localization of the cause of endogenous hyperinsulinemic hypoglycemia using 3 various imaging modalities. We have also documented the pattern of weight loss after removal of the pancreatic tumor, new information that this case report adds to our knowledge of this condition.

\section{CASE}

A 34-year-old female had an episode of loss of consciousness 10 months prior to admission. During that time, she was brought to an emergency room and was found to have low blood sugar levels. She regained consciousness after intravenous glucose administration. No other diagnostic work-ups were done at that time and she was discharged.

Since then, she has had recurrent episodes of dizziness, disorientation, incoherence, hunger pangs, cold sweats, palpitations and tremors. She developed the frequent

e-ISSN 2308-118X

Printed in the Philippines

Copyright $(2015$ by the JAFES

Received: January 30, 2015. Accepted: August 13, 2015.

http://dx.doi.org/10.15605/jafes.030.02.05 urge to eat and she learned to avoid these symptoms by eating every 2 hours. She progressively gained weight as a result.

She denies taking any oral hypoglycemic agent or administering insulin. She has no known illnesses. She is gravida 8 para 2 (2-0-6-2)

Seven months prior to admission she consulted an endocrinologist. Hyperinsulinemic hypoglycemia was considered. A supervised fast was done. When she developed symptoms of hypoglycemia, venous blood was taken and sent for glucose and insulin levels. Serum glucose was low at $2.87 \mathrm{mmol} / \mathrm{L}(52.1 \mathrm{mg} / \mathrm{dL})$ while the serum insulin was inappropriately unsuppressed at 166.3 pmol/L (normal: 17.8 - 173.0).

Abdominal CT scan did not show any focal lesion in the pancreas. A subsequent abdominal MRI also did not show any focal pancreatic lesion.

With the persistence of symptoms, the patient consulted our institution for further work-up.

Physical examination revealed an obese female with a weight of $107 \mathrm{~kg}$, height of $152 \mathrm{~cm}$ and body mass index of $46.31 \mathrm{~kg} / \mathrm{m}^{2}$. Her ideal body weight is $46 \mathrm{~kg}$ based on the

Corresponding author: Mark Anthony Sandoval, MD

Section of Endocrinology, Diabetes and Metabolism

Medical Research Laboratory, $2^{\text {nd }}$ floor, Philippine General Hospital

Taft Avenue, Manila, 1000, Philippines

Tel. No.: +632-5548400 local 3230

E-mail: markanthony_sandoval@yahoo.com 
Broca-Devine formula. Vital signs were normal. She was conversant and coherent at the time of examination. There was no acne, hirsutism, violaceus striae nor ecchymoses. There was no goiter. The rest of the PE findings are unremarkable.

A repeat supervised fast was done. Within four hours after the last food intake, the patient developed hypoglycemic symptoms. Capillary blood glucose was 2.5 $\mathrm{mmol} / \mathrm{L}(45 \mathrm{mg} / \mathrm{dL})$. Venous blood was extracted and sent for serum glucose, insulin, C-peptide and cortisol. Serum glucose was low at $3.8 \mathrm{mmol} / \mathrm{L}(68.83 \mathrm{mg} / \mathrm{dL})$ while insulin and C-peptide were both elevated [serum insulin 25.13 $\mathrm{uIU} / \mathrm{mL}$ (normal: 4.50 - 20.00); C-peptide $3.60 \mathrm{nmol} / \mathrm{L}$ (normal: 0.35 - 1.17)]. Serum cortisol was $253.83 \mathrm{nmol} / \mathrm{L}$ (normal: $160-620$ ).

Endoscopic ultrasound revealed a $1.5 \times 2.0 \mathrm{~cm}$ hypoechoic to isoechoic mass at the head of the pancreas (Figure 1). No increased vascularity was noted. There were no

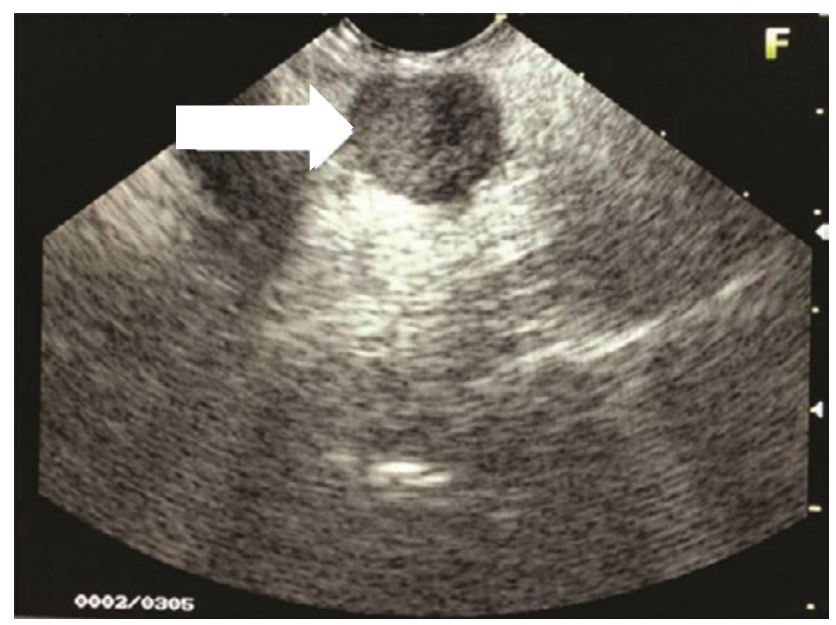

Figure 1. Endoscopic ultrasound shows a $1.5 \times 2.0 \mathrm{~cm}$ hypoechoic to isoechoic mass at the head of the pancreas.

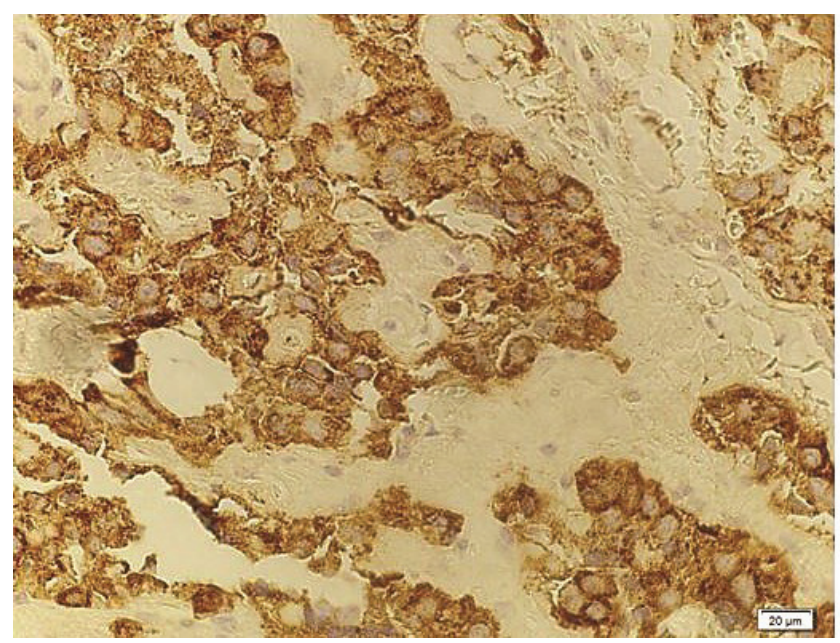

peripheral lymphadenopathies. The liver was free of any metastasis but appeared diffusely hyperechoic.

On exploratory laparotomy, a 2 × $2 \mathrm{~cm}$ well-circumscribed pancreatic head mass was identified. Intraoperative ultrasound revealed that the mass was more than $3 \mathrm{~mm}$ away from the pancreatic duct and it was enucleated. In addition, elective cholecystectomy and bilateral tubal ligation were also performed per patient's preference. Histopathologic examination using H\&E stain revealed an islet cell tumor (Figure 2), $2 \mathrm{~cm}$ in greatest diameter. Immunohistochemical staining with chromogranin and synaptophysin (Figure 3) are both positive, confirming the neuroendocrine nature of the tumor. Immunohistochemical staining for insulin would be able to morphologically demonstrate that the tumor produces insulin but this test is not available in the Philippines.

Post-operatively, there was no more recurrence of hypoglycemic symptoms and all capillary glucose levels were normal.

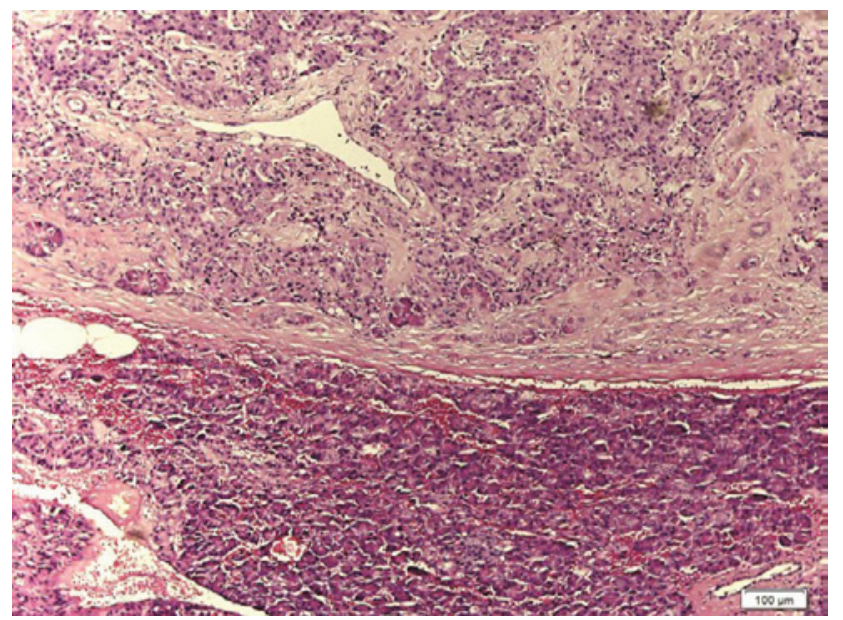

Figure 2. Photomicrograph showing a pancreatic neuroendocrine tumor in the upper area separated from the normal pancreatic tissue below by a fibrous capsule in the middle (H\&E stain, $x$ 100).

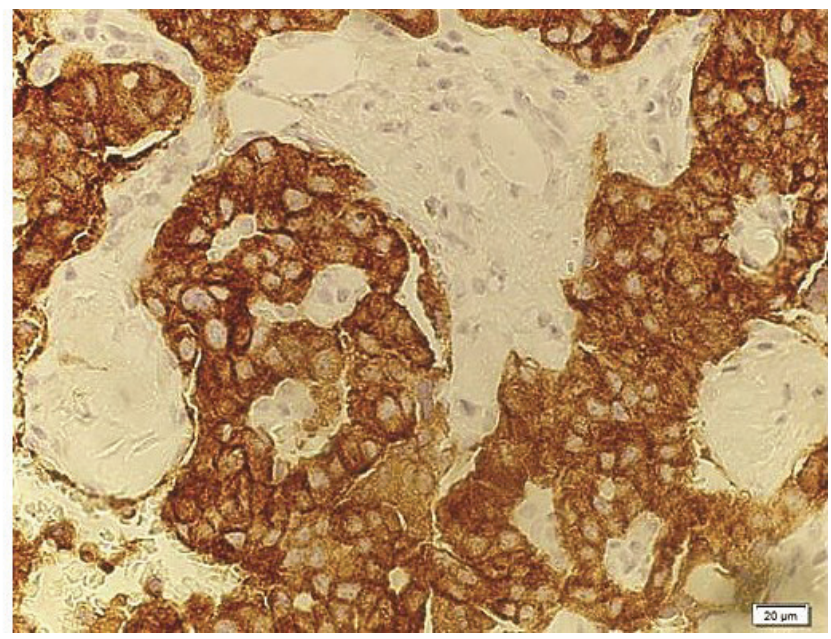

Figure 3. Immunohistochemical staining for synaptophysin (left) and chromogranin (right) are both positive among the neoplastic cells, confirming the neuroendocrine nature of the tumor ( $x 400)$. 
After 8 weeks, there was no recurrence of hypoglycemic symptoms. The patient can now tolerate 8.5 hours without food intake and does not experience any hypoglycemic symptoms. Plasma glucose taken after 8.5 hours of fasting was $6.1 \mathrm{mmol} / \mathrm{L}(110 \mathrm{mg} / \mathrm{dL})$, with a serum insulin level of $22 \mathrm{uIU} / \mathrm{mL}$ and serum C-peptide at $1.53 \mathrm{nmol} / \mathrm{L}$.

She also lost a significant amount of weight, losing $18 \mathrm{~kg}$ within 8 weeks after surgery (Figure 4). We have documented the pattern of weight loss shown in Figure 5. Weight was first monitored at the $6^{\text {th }}$ post-operative day when the patient did not have any intravenous and urethral catheters in place and when she was already comfortably ambulating with minimal pain. The weight was documented daily until the $56^{\text {th }}$ post-operative day. It appears that weight loss was not linear. There was a gradual weight loss during post-op day 6 to day 19 (3 kg over 13 days; average of $0.23 \mathrm{~kg} / \mathrm{d}$ ). But from post-op day 19 to day 39, there was a rapid weight reduction. She lost $16 \mathrm{~kg}$ in this span of 20 days (average of $0.80 \mathrm{~kg} / \mathrm{d}$ ). Subsequently, there was no more rapid weight loss and her weight was maintained within the range of $88-89 \mathrm{~kg}$ until the 56th post-operative day.

Day 6 post-op (107 kg)
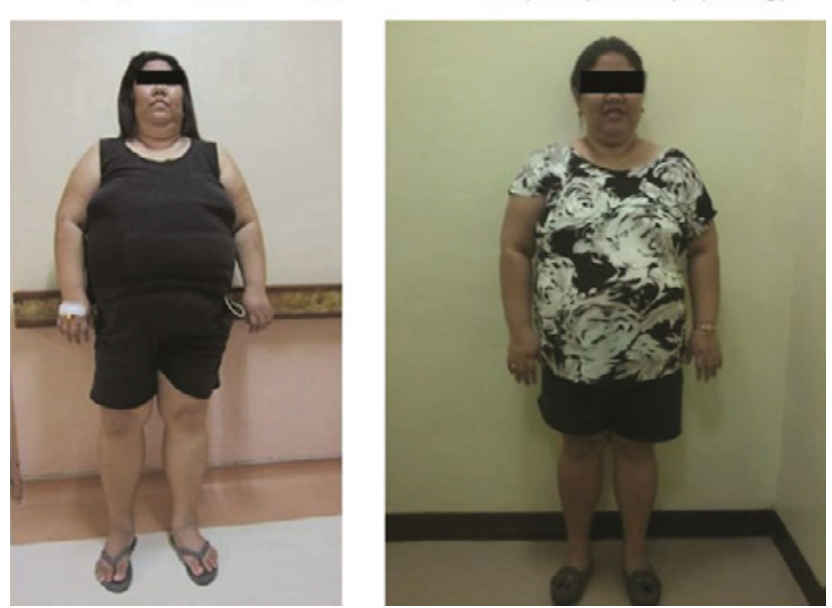

Figure 4. Appearance of the patient at day 6 post-op and at day 56 post-op.

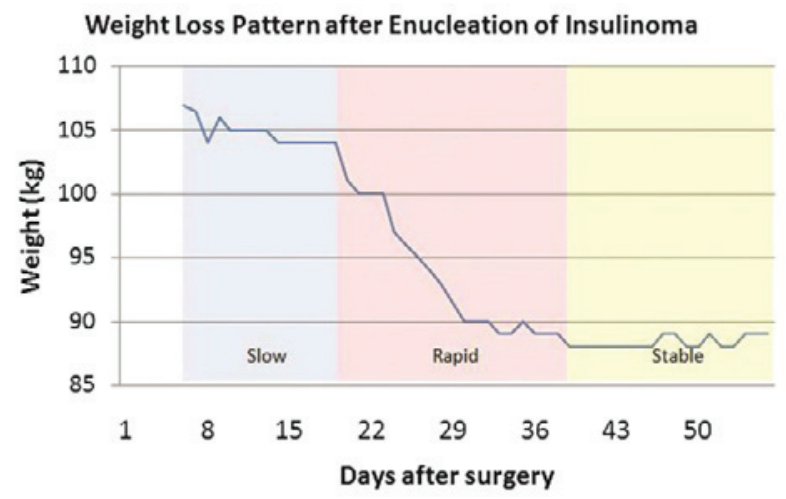

Figure 5. Weight loss velocity graph demonstrating an initial gradual weight loss followed by a rapid loss of weight and eventual stabilization of the patient's weight.
A recall of her 24-hour food intake before and after surgery showed that she consumed much fewer calories after surgery. Prior to surgery, her usual daily intake was as follows: 3,687 kcal comprised of $519 \mathrm{~g}$ carbohydrates, 70 $\mathrm{g}$ proteins and $135 \mathrm{~g}$ fats. After the surgery, her usual daily intake was 1,284 kcal comprised of $132 \mathrm{~g}$ carbohydrates, $34 \mathrm{~g}$ proteins and $66 \mathrm{~g}$ fats.

Levels of physical activity before and after surgery were similar.

\section{DISCUSSION}

This patient had symptoms of hypoglycemia, documented low capillary blood glucose levels and resolution of symptoms after the administration of intravenous glucose or food intake. Since the patient already had hypoglycemic symptoms when the capillary blood glucose was determined to be $45 \mathrm{mg} / \mathrm{dL}$, we did not repeat the 72-hour fast even if the serum glucose was $68.83 \mathrm{mg} / \mathrm{dL}$.

After the initial episode, there was no recurrence of syncope since the patient learned to eat larger quantities of food frequently to avoid the symptoms of hypoglycemia, but at the expense of weight gain.

The cause of the hypoglycemia was found to be due to high insulin levels since the measured plasma insulin levels were elevated despite the low level of plasma glucose. The finding of an elevated C-peptide level suggests an endogenous source of the excess insulin. Thus, the combination of low glucose, high insulin and high C-peptide levels is consistent with a diagnosis of endogenous hyperinsulinemic hypoglycemia.

The increased frequency and amount of food intake plus the anabolic effects of insulin both contributed to the patient's significant weight gain.

Differentials of endogenous hyperinsulinemic hypoglycemia include an insulin-producing PNET, sulfonylurea intake, insulin autoantibodies and nesidioblastosis. A PNET is the most common of these causes. Sulfonylurea intake can be determined by history, which the patient denied. Had we suspected surreptitious intake, we could have tested for sulfonylurea in the urine. However, this test is not available in the Philippines.

Insulin-producing tumors are almost always located in the pancreas and imaging tests are done to localize them and plan the necessary surgery to remove them. ${ }^{2} \mathrm{CT}$ scan and MRI are typically the first imaging tests ordered to visualize such tumors. However, the sensitivity of CT scan is only $78 \%$ and that of MRI, $71 \%$, according to a study from Thailand. ${ }^{3}$ Endoscopic ultrasound (EUS) has a greater sensitivity at $93 \% .^{3}$ For our patient, both CT scan and MRI failed to visualize the tumor but it was eventually localized by EUS. There are authors who state that since EUS can detect all tumors by every other 
conventional technique, there is no need for the other imaging tests. ${ }^{4}$

Our patient recovered clinically after the surgery. Her two main symptoms - hypoglycemia and weight gain - have resolved. There was no recurrence of hypoglycemic symptoms despite no food intake for 8.5 hours. Both her insulin and C-peptide levels were already within acceptable values for the prevailing level of serum glucose after surgery.

The patient was also very satisfied with the resultant weight loss. The weight loss was due to decreased food intake as she no longer had to eat frequently to avoid hypoglycemic symptoms. A recall of her food intake for a 24-hour period showed that her total calorie intake decreased after surgery. Among the macronutrients, it was the carbohydrates that showed the greatest reduction in intake: $75 \%$ reduction for carbohydrates, 51\% reduction for proteins and $51 \%$ reduction in fats. Increase in the level of physical activity does not seem to have played a role in her weight loss since she mentioned that her level of physical activity did not change before and after the surgery.

There were case reports that mentioned the amount of weight loss over a specified amount of time after surgery but have not documented the daily change in weight over that period of time. Madathil has reported a $6 \mathrm{~kg}$ weight loss over a span of 6 weeks in their patient, while Lemoncito reported a $17 \mathrm{~kg}$ weight loss over one month. 5,6 Both however, did not show the daily weight change. A prospective study that followed up 36 patients who underwent surgery for insulinoma did not include weight change as an outcome as well. ${ }^{7}$ We have not come across any case report which meticulously documented the daily pattern of weight loss after removal of an insulin-secreting PNET and this is novel information that this case report contributes.

The pattern of weight loss being described here may not necessarily be generalizable to all patients. Meticulous measurement of weight on a daily basis may be done on other patients to see if there is a similar pattern. Knowing the pattern of weight loss may be important to advise patients what to expect after surgery. For this specific patient, she was particularly concerned about her weight gain from the illness and was expecting to lose the excess weight after the operation. If the pattern documented in this case report is true for all patients, then doctors can advise their patients to expect that rapid weight loss occurs on the $19^{\text {th }}$ to $39^{\text {th }}$ day after surgery and that no further weight loss occurs afterwards. However, it is not yet known if further weight loss occurs after the $56^{\text {th }}$ day post-op since the observation in this patient was only until this period.

\section{CONCLUSION}

The combination of loss of consciousness, hypoglycemic symptoms and weight gain should raise the suspicion of hyperinsulinemic hypoglycemia. Absence of any focal pancreatic lesions on abdominal CT scan and MRI should not rule out the presence of a pancreatic mass. Endoscopic ultrasound is a more sensitive test to localize these lesions. Surgical removal of the insulin-producing pancreatic neuroendocrine tumor resolves the hypoglycemia and leads to weight loss. The pattern of weight loss after removal of the functioning tumor was documented in this case, with a slow weight loss initially followed by a phase of rapid weight loss.

\section{Acknowledgements}

We thank the Department of Pathology of the College of Medicine, University of the Philippines Manila for the photomicrographs; and nutritionist Ms. Ellesmere Gaille Bacabac, $\mathrm{RND}$, for the conversion of the food recall data into the pertinent nutritional information.

\section{References}

1. Klimstra DS, Modlin IR, Coppola D, Lloyd RV and Suster S. The pathologic classification of neuroendocrine tumors. Pancreas. 2010;39(6):707-712

2. Vaidakis D, Karoubalis J, Papa T, Pladitis G and Zografos GN Pancreatic insulinoma: Current issues and trends. Hepatobiliary and Pancreatic Diseases International. 2010; 9:234-241.

3. Pongprasobchai S, Lertwattanarak R, Pausawasdi N, Prachayakul V. Diagnosis and localization of insulinoma in Thai patients: Performance of endoscopic ultrasonography compared to computed tomography and magnetic resonance imaging. J Med Assoc Thai. 2013 Feb;96(Suppl 2):S187-93.

4. Kaltsas GA, Besser GM, Grossman AB. The diagnosis and medical management of advanced neuroendocrine tumors. Endocrine Reviews. 2004;25(3):458-511. http://dx.doi.org/10.1210/er.2003-0014.

5. Madathil A and Weaver J. Insulinoma presenting as postprandial hypoglycemia. BMJ Case Reports. 2011. http://dx.doi.org/10.1136/ bcr.07.2011.4477.

6. Lemoncito MV, Josol CV, Ramos HC, Quimpo JA, Lantion-Ang FL, Guazon MLV. Recurrent hyperinsulinemic hypoglycemia in a 23year old male with negative imaging studies: An enigma of insulinoma. Philipp J Intern Med. 2011;49(3):177-184.

7. Tsang YP, Lang BH, Shek TW. Assessing the short- and long-term outcomes after resection of benign insulinoma. ANZ Journal of Surgery 2014 Oct 23. doi: 10.1111/ans.12891.

Articles and any other material published in the JAFES represent the work of the author(s) and should not be construed to reflect the opinions of the Editors or the Publisher. Authors are required to accomplish, sign and submit scanned copies of the JAFES Declaration: that the article represents original material, that is not being considered for publication or has not been published or accepted for publication elsewhere. Consent forms, as appropriate, have been secured for the publication of information about patients; otherwise, authors declared that all means have been exhausted for securing such consent. The authors have signed disclosures that there are no financial or other relationships that might lead to a conflict of interest. All authors are required to submit Authorship Certifications that the manuscript has been read and approved by all authors, and that the requirements for authorship have been met by each author. 